\title{
HAMILTONIAN SYSTEMS IN A NEIGHBORHOOD OF A SADDLE POINT
}

BY

VIOREL BARBU

\begin{abstract}
The behavior of Hamiltonian differential systems associated with a concave convex function $H$ in a Hilbert space is studied by variational methods. It is shown that under quite general conditions on the function $H$ the system behaves in a neighborhood of a minimax saddle point of $H$ much like as in the classical theory of ordinary differential systems. The results extend previous work of R. T. Rockafellar.
\end{abstract}

1. Introduction. Let $E$ be a real Hilbert space with norm $|\cdot|$ and inner product (, ). Let $H: E \times E \rightarrow[-\infty,+\infty]$ be an extended real valued concave-convex function on $E \times E$. The symbol $\partial H(x, p)=\left(-\partial_{x} H(x, p)\right.$, $\left.\partial_{p} H(x, p)\right)$ denotes the set of all subgradients of $H$ at the point $(x, p)$, i.e.,

$$
\begin{aligned}
-\partial_{x} H(x, p) & =\{v \in E ; H(x, p)>H(y, p)-(x-y, v) \text { for all } y \in E\}, \\
\partial_{p} H(x, p) & =\{w \in E ; H(x, p) \leqslant H(x, q)+(p-q, w) \text { for all } q \in E\} .
\end{aligned}
$$

The operator (which in general is multivalued) $\partial H: E \times E \rightarrow E \times E$ is called the subdifferential of $H$. If $H$ is Gâteaux differentiable with respect to $x$ and $p, \partial H(x, p)$ reduces to the gradient $\nabla H(x, p)$ of $H$ at $(x, p)$.

In this paper we shall study the differential system

$$
\begin{array}{ll}
x^{\prime}(t) \in \partial_{p} H(x(t), p(t)), & \text { a.e. } t>0, \\
p^{\prime}(t) \in-\partial_{x} H(x(t), p(t)), & \text { a.e. } t>0,
\end{array}
$$

in the neighborhood of a saddle point $(\tilde{x}, \tilde{p})$ of $H$. By a solution to (1.1) over $[0,+\infty[$ we shall mean a pair of absolutely continuous functions $x:[0$, $+\infty[\rightarrow E$ and $p:[0,+\infty[\rightarrow E$ such that (1.1) is satisfied for almost all $t>0$ (we have denoted by ' the differentiation symbol $d / d t$ ).

Such a system is called a generalized Hamiltonian system and it arises for example in the characterisation of optimal arcs in the convex problem of Lagrange (see e.g. [1])

$$
\min \left\{\int_{0}^{T} L\left(x(t), x^{\prime}(t)\right) d t ; x(0)=x_{0}\right\} .
$$

Received by the editors February 23, 1977 and, in revised form, June 20, 1977.

AMS (MOS) subject classifications (1970). Primary 34G05, 47Hxx, 47H15, 49Axx, 49A10.

Key words and phrases. Hamiltonian systems, concave-convex. function, saddle point, subdifferential, Lagrange problem, Euler-Lagrange equations. 
Here $L$ is the Lagrangian function corresponding to $H$, i.e., $L(x, u)=$ $\sup \{(p, u)-H(x, p) ; p \in E\}$ for all $x \in E$ and $u \in E$.

It is well known that every saddle point $(\tilde{x}, \tilde{p})$ of function $H$ satisfies the equation $(0,0) \in \partial H(\tilde{x}, \tilde{p})$. This fact says that $(\tilde{x}, \tilde{p})$ is a stationary solution of the system (1.1). Proceeding as in [9] we shall make a translation, so that the saddle point $(\tilde{x}, \tilde{p})$ appears at the origin. Specifically, we define

$$
\tilde{H}(x, p)=H(x+\tilde{x}, p+\tilde{p})-H(\tilde{x}, \tilde{p})
$$

and observe that $H$ is concave-convex and satisfies $(0,0) \in \partial \tilde{H}(0,0), \tilde{H}(0,0)$ $=0$. It should be observed also that the solutions to the given system (1.1) are the translates by $(\tilde{x}, \tilde{p})$ of the solutions to

$$
\left(p^{\prime}(t), x^{\prime}(t)\right) \in \partial \tilde{H}(x(t), p(t)) \text {, a.e. } t>0 \text {. }
$$

In this way the problem is reduced to the study of the Hamiltonian system (1.1) in a neighborhood of $(0,0)$. Moreover, we may assume that

$$
(0,0) \in \partial H(0,0), \quad H(0,0)=0,
$$

or, equivalently,

$$
H(x, 0) \leqslant H(0,0)=0 \leqslant H(0, p) \text { for all } x \in E, p \in E .
$$

In a recent paper [9] (see also [10]) R. T. Rockafellar investigated the existence of solutions $(x(t), p(t))$ tending to 0 as $t \rightarrow+\infty$ or as $t \rightarrow-\infty$ in the case of a finite dimensional differential system of the form (1.1). Theorem 1 below extends these results to Hamiltonian systems (1.1) defined on the Hilbert space $E$.

Now we proceed to formulate the main results precisely. The following conditions on $H$ will be considered.

(a) The function $H(x, p)$ is concave and upper semicontinuous in $x$ for each $p$ and convex and lower semicontinuous in $p$ for each $x$.

(b) There exists $\eta>0$ such that

$$
-\infty<H(x, p)<+\infty \text { for }|x|<\eta,|p|<\eta .
$$

Furthermore, $H$ is strictly concave in $x$ and strictly convex in $p$ for $|x|<\eta$ and $|p| \leqslant \eta$.

(c) $(0,0) \in \partial H(0,0)$ and $H(0,0)=0$.

(d) For each $\varepsilon>0$ there exist positive constants $\eta_{i}, i=1,2$, such that $|H(x, p)| \leqslant \varepsilon|p|$ for $|x| \leqslant \eta_{1}$ and $|p|<\eta_{2}$.

(e) For each $r \in] 0, \eta[, \inf \{H(0, p) ;|p|=r\}>0$ and $\inf \{-H(x, 0) ;|x|=$ $r\}>0$.

It should be recalled that (1.5) implies that the operator (multivalued) $\partial H$ is locally bounded on $\Sigma_{\eta}=\{(x, p) \in E \times E ;|x|<\eta,|p|<\eta\}$. Thus choosing $\eta$ sufficiently small we may assume that $\partial H$ is bounded on $\Sigma_{\eta}$, i.e. there exists a positive constant $C$ such that 


$$
\begin{aligned}
\|\partial H(x, p)\|=\sup \left\{\left|y_{1}\right|+\left|y_{2}\right| ;\left(y_{1}, y_{2}\right) \in \partial H(x, p)\right\} & <C \\
& \text { for all }(x, p) \in \Sigma_{\eta} .
\end{aligned}
$$

Finally, it should be observed that if $E$ is finite dimensional then condition (e) is implied by the strict concavity-convexity of $H$.

Let $\Gamma$ denote the set of all pairs $\left(x_{0}, p_{0}\right) \in E \times E$ such that there is a solution to (1.1) over $[0,+\infty$ [ satisfying

$$
\begin{gathered}
x(0)=x_{0}, \quad p(0)=p_{0} ; \\
\lim _{t \rightarrow+\infty} x(t)=0, \quad \lim _{t \rightarrow+\infty} p(t)=0 .
\end{gathered}
$$

The subset $\Gamma \subset E \times E$ may be viewed as a multivalued (in general) operator from $H$ into itself. Theorem 1 below gives a detailed description of $\Gamma$ in a neighborhood of the origin.

THEOREM 1. There is a neighborhood $U \times V$ of $(0,0)$ (which can be chosen arbitrarily small) such that:

(i) $U$ is open and $\Gamma$ is one-to-one and demicontinuous (i.e. strongly-weakly continuous) from $U$ onto $V$.

(ii) There exists a continuous convex function $\Psi$ which is Gâteaux differentiable on $U$ such that

$$
\Gamma x_{0}=-\nabla \Psi\left(x_{0}\right) \text { for all } x_{0} \in U .
$$

(iii) For each $\left(x_{0}, p_{0}\right) \in \Gamma \cap(U \times V)$ the solution $(x(t), p(t))$ to (1.1) over $[0,+\infty[$ satisfying (1.7) and (1.8) is unique and remains in $\Gamma \cap(U \times V)$.

(iv) $\Gamma$ is a solution of the operator equation

$$
H\left(x_{0},-\Gamma x_{0}\right)=0 \text { for all } x_{0} \in U .
$$

(v) If among the solutions to (1.1) over $[0,+\infty[$ there is one, $(x(t), p(t))$, which remains in $U \times V$ for all $t \geqslant 0$, then it also satisfies (1.8), i.e. $p(t)=$ $\Gamma x(t)$ for all $t \geqslant 0$.

This theorem can be used to derive similar results for behavior over the interval ] $-\infty, 0]$. To this purpose it suffices to apply the theorem to the Hamiltonian function $H(x, p)=H(x,-p)$. Thus we may conclude that under the above assumptions the behavior of (1.1) in the neighborhood of a stationary point closely resembles that of a classical saddle point in the theory of ordinary differential systems.

It should be also observed that for $U \times V$ sufficiently small, (1.6) shows that each solution $(x(t), p(t))$ to (1.1) emanating from $\left(x_{0}, p_{0}\right) \in U \times V$ satisfies

$$
\left|x^{\prime}(t)\right| \leqslant C, \quad\left|p^{\prime}(t)\right| \leqslant C \quad \text { a.e. } t>0 .
$$

Finally, we notice that part (v) of Theorem 1 can be interpreted as an implicit function theorem for the concave-convex function $H$. 
The plan of the paper is the following In $\$ 2$ we shall develop for later use some facts concerning the Lagrange problem assoc, ated with (1.1). These will enable us, in $\$ 3$, to conclude the proof of Theorem 1 .

We assume familiarity with basic concepts and results on convex analysis and theory of concave-convex functions on infinite dimensional spaces (a detailed account of this theory may be found in [7] and [8]).

2. The Lagrange problem associated with system (1.1). Let $L_{0}: E \times E \rightarrow$ ] $-\infty,+\infty$ ] be the Lagrangian function defined by

$$
L_{0}(x, u)=\sup \{(p, u)-H(x, p) ;|p| \leqslant \eta\} .
$$

It is well known that $L_{0}$ is convex and lower semicontinuous on the product space $E \times E$ and

$$
H(x, p)=\sup \left\{(p, u)-L_{0}(x, u) ; u \in H\right\} \quad \text { for }|p| \leqslant \eta \text { and all } x \in E \text {. }
$$

LEMMA 1. Under assumptions (a)-(e) the following relations hold:

$$
\begin{gathered}
0=L_{0}(0,0) \leqslant \\
L_{0}(x, u)<+\infty \text { for all }|x| \leqslant \eta \text { and } u \in E, \\
\lim _{|u| \rightarrow 0} L_{0}(0, u) /|u|=0 .
\end{gathered}
$$

Proof. We have from conjugacy formula (2.1) that

$$
L_{0}(x, u) \geqslant-H(x, 0) \text { for all } x \in E, u \in E .
$$

On the other hand $H(x, 0) \leqslant 0$ because $(0,0)$ is a saddle point of $H$ (see (1.4)). This fact implies that $L_{0}(0,0)=\sup \{-H(0, p) ;|p|<\eta\}=0$ as claimed. As mentioned earlier, condition (b) (part (1.5)) implies (1.6). From the latter it can be easily derived that the function $H(x, p)$ is Lipschitzian on $\Sigma_{\eta}$. In particular, we infer that $H$ is bounded on $\Sigma_{\eta}$. This fact combined with (2.2) yields

$$
L_{0}(x, u) \leqslant \eta|u|+M \text { for all }|x| \leqslant \eta \text { and } u \in E,
$$

where $M$ is independent of $x$ and $u$. To prove (2.5) we proceed as follows. By (2.1) it follows that for every $u$ the infimum defining $L_{0}(0, u)$ is attained in a (unique) point $p_{u}$. Hence we have

$$
L_{0}(0, u)=\left(p_{u}, u\right)-H\left(0, p_{u}\right) \text { for all } u \in E .
$$

Since the function $L_{0}(0, \cdot)$ is continuous at the origin and $L_{0}(0,0)=0$, it follows from (2.7) that $\lim _{|u| \rightarrow 0} H\left(0, p_{u}\right)=0$ (we note that $\left.\left|p_{u}\right|<\eta\right)$. Thus (e) implies that

$$
\lim _{|u| \rightarrow 0} p_{u}=0
$$


Then again using (2.7) we get (2.5), thereby completing the proof of Lemma 1.

For a reason which will be clear later, it will be more convenient to replace the function $L_{0}$ by

$$
L(x, u)= \begin{cases}L_{0}(x, u) & \text { if }|x| \leqslant \eta \text { and }|u| \leqslant \eta \\ +\infty & \text { otherwise }\end{cases}
$$

The function $L$ is likewise $L_{0}$, convex and lower semicontinuous on $E \times E$. Thus the Hamiltonian function $H_{1}: E \times E \rightarrow[-\infty,+\infty]$,

$$
H_{1}(x, p)=\sup \{(p, u)-L(x, u) ; u \in E\}
$$

is concave-convex and closed on $E \times E$ (see [7], [8]). The lemma below shows that the functions $H$ and $H_{1}$ agree on a neighborhood of $(0,0)$.

LeMma 2. There is $0<\delta<\eta$ such that

$$
H(x, p)=H_{1}(x, P) \text { for }|x| \leqslant \delta,|p|<\delta .
$$

Proof. For each $x \in E$ and $p \in E$ the supremum defining $H(x, p)$ is attained in a point $u \in \partial_{p} H(x, p)$. Hence to prove (2.11) it suffices to show that for $|x|$ and $|p|$ sufficiently small one has (see (2.2) and (2.9))

$$
\sup \left\{|y| ; y \in \partial_{p} H(x, p)\right\}<\eta \text {. }
$$

To this end we consider $y \in \partial_{p} H(x, p)$ arbitrary but fixed. By definition of $\partial_{p} H(x, p)$ we have

$$
(y, p-\rho w) \geqslant H(x, p)-H(x, \rho w) \text { for all }|w|=1,
$$

where $\rho$ is positive and sufficiently small. Setting $w=y /|y|$ one sees that for all $|p| \leqslant \rho / 2$ and $|x| \leqslant \eta$ one has

$$
|y| \leqslant(H(x, \rho w)-H(x, p)) / 2 \rho .
$$

Thus applying (d) we deduce that $|y|<\eta$ for all $x, p \in E$ with $|x|<\delta$ and $|p| \leqslant \delta$ where $\delta$ is suitably chosen. This completes the proof.

Let $\Psi: E \rightarrow[0,+\infty[$ be the function defined by

$$
\Psi\left(x_{0}\right)=\inf \left\{\int_{0}^{\infty} L\left(y(t), y^{\prime}(t)\right) d t ; y(0)=x_{0}\right\}
$$

where the infimum is over the class of all absolutely continuous arcs $y$ :

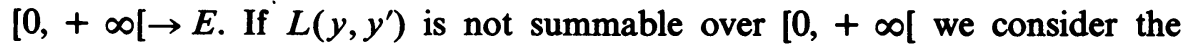
integral in (2.13) to have the value $+\infty$ by convention. The convexity of $L$ implies the convexity of $\Psi$ on $E$. Let $D(\Psi)$ be the effective domain of $\Psi$, i.e.,

$$
D(\Psi)=\left\{x_{0} \in E ; \Psi\left(x_{0}\right)<+\infty\right\} .
$$

We next prove under assumptions (a)-(e) several results for the function $\Psi$ that will be used ultimately to prove Theorem 1 . 
Lemma 3. One has

$$
D(\Psi)=\left\{x_{0} \in E ;\left|x_{0}\right| \leqslant \eta\right\}=S_{\eta} .
$$

Moreover, for each $x_{0} \in S_{\eta}$ the infimum defining $\Psi\left(x_{0}\right)$ is attained in a unique arc $x(t)$ satisfying

$$
\lim _{t \rightarrow \infty} x(t)=0 .
$$

Proof. Let $x_{0}$ be fixed in $S_{\eta}$. Then $y(t)=x_{0} \exp (-t)$ is a feasible arc in problem (2.13). In fact since $L_{0}(0,0)=0$ and $\partial L_{0}$ is locally bounded on $\Sigma_{\eta}$ (see Lemma 1), it follows that $L\left(y, y^{\prime}\right) \in L^{1}(0, \infty)$. Therefore, $S_{\eta} \subset D(\Psi)$. The justification of the converse inclusion is seen by (2.9). Let $x_{0} \in D(\Psi)$ be fixed and let $\left\{x_{n}\right\}$ be a sequence of absolutely continuous functions over $[0,+\infty[$ such that

$$
\begin{gathered}
\left|x_{n}(t)\right| \leqslant \eta, \quad\left|x_{n}^{\prime}(t)\right| \leqslant \eta \quad \text { a.e. } t>0, \\
\Psi\left(x_{0}\right) \leqslant \int_{0}^{\infty} L_{0}\left(x_{n}(t), x_{n}^{\prime}(t)\right) d t \leqslant \Psi\left(x_{0}\right)+1 / n .
\end{gathered}
$$

Extracting a subsequence, if necessary, we may assume that

$$
\begin{array}{ll}
x_{n} \rightarrow x & \text { weakly in } L^{\infty}(0, \infty ; E), \\
x_{n}^{\prime} \rightarrow x^{\prime} & \text { weakly in } L^{\infty}(0, \infty ; E) .
\end{array}
$$

We then have

$$
|x(t)| \leqslant \eta, \quad\left|x^{\prime}(t)\right| \leqslant \eta \quad \text { a.e. } t>0 .
$$

On the other hand, it is well known that for every $T>0$ the function $(y, v) \rightarrow \int_{0}^{T} L_{0}(y(t), v(t))$ is convex and lower semicontinuous (equivalently, weakly lower semicontinuous) on $L^{\infty}(0, T ; E) \times L^{\infty}(0, T ; E)$. This fact combined with (2.15)-(2.17) yields

$$
\Psi\left(x_{0}\right)=\int_{0}^{\infty} L\left(x(t), x^{\prime}(t)\right) d t .
$$

To prove the uniqueness of $x(t)$ it suffices to observe that in view of (b) and (2.1) the function $L_{0}$ is strictly convex on $\Sigma_{\eta}$.

By using (2.6) it follows that $-H(x(t), 0) \in L^{1}(0, \infty)$. Inasmuch as the function $x \rightarrow H(x, 0)$ is continuous on $S_{\eta}$ and $x(t)$ is uniformly continuous over $\left[0,+\infty\left[\right.\right.$ (because $\left|x^{\prime}(t)\right| \leqslant \eta$, a.e. $t>0$ ), we deduce that $\lim _{t \rightarrow \infty} H(x(t)$, $0)=0$. Then it follows from (e) that $\lim _{t \rightarrow \infty} x(t)=0$ as claimed.

LEMMA 4. The function $\Psi$ is continuous on $S_{\eta}=\left\{x_{0} \in E ;\left|x_{0}\right|<\eta\right\}$ and for every $\sigma \in] 0, \eta[$ there exists $\rho>0$ such that

$$
\left\{x_{0} \in E ; \Psi\left(x_{0}\right)<\rho\right\} \subset S_{\sigma} .
$$

Proof. Since $\Psi$ is convex and finite on $S_{\eta}$, to prove that $\Psi$ is continuous on $S_{\eta}$ it suffices to show that it is lower semicontinuous on this subset. But this 
follows by a standard argument involving Fatou's lemma, which we do not reproduce here. To prove the second part of this lemma we set $\chi(x)=$ $-H(x, 0)$ and fix $x_{0}$ in $D(\Psi)=S_{\eta}$. Let $x$ be the unique arc which yields the minimum in the definition of $\Psi\left(x_{0}\right)$. We have (see e.g. [2, p. 189])

$$
\frac{d}{d t} \chi(x(t))=\left(B x(t), x^{\prime}(t)\right) \text { a.e. } t>0,
$$

where $B x(t)$ is any measurable section of $\partial \chi(x(t))$. Since $\partial \chi$ is locally bounded (in fact it is bounded) on $S_{\eta}$ we get that

$$
\left|\frac{d}{d t} \chi(x(t))\right|<M \quad \text { a.e. } t>0,
$$

where $M$ is a positive constant independent of $x_{0}$. Observe that this inequality combined with the obvious relation

$$
\Psi\left(x_{0}\right)>\int_{0}^{\infty} \chi(x(t)) d t
$$

entail

$$
T \chi\left(x_{0}\right)<\Psi\left(x_{0}\right)+M T^{2} \text { for all } T>0
$$

and, hence,

$$
\left|x_{0}\right| \leqslant \omega^{-1}\left(\Psi\left(x_{0}\right) / T+M T\right) \text { for all } T>0,
$$

where $\omega:[0,+\infty[\rightarrow[0,+\infty[$ is defined by

$$
\omega(r)=\inf \{x(x) ;|x|=r\} .
$$

The function $\omega$ is continuous, monotone increasing and $\omega(0)=0$. Furthermore, (e) implies that $\lim _{r \rightarrow 0} \omega^{-1}(r)=0$ and (2.19) thus implies that $\left|x_{0}\right|<\sigma$ if $\Psi\left(x_{0}\right)<\rho$ ( $\rho$ suitably chosen) as aimed.

It is convenient for later purposes to reduce problem (2.13) to a variational problem on a finite interval.

Lemma 5. Let $x_{0} \in D(\Psi)$ and let $x$ be the unique arc which yields the minimum in $\Psi\left(x_{0}\right)$. Then for every $T>0, x$ is the unique solution of the minimization problem

$$
\inf \left\{\int_{0}^{T} L\left(y(t), y^{\prime}(t)\right) d t+\Psi(y(T)) ; y(0)=x_{0}\right\} .
$$

Furthermore, the value of (2.20) is $\Psi\left(x_{0}\right)$ and

$$
\Psi(x(T))=\int_{T}^{\infty} L_{0}\left(x(t), x^{\prime}(t)\right) d t \text { for all } T>0 .
$$

Proof. Let $y$ be any feasible arc in (2.20). Then $|y(t)|<\eta$ for all $t>0$ and, hence, by Lemma 3 the infimum defining $\Psi(y(T))$ is attained in a unique $\operatorname{arc} z(t)$. In other words, 


$$
\Psi(y(T))=\int_{0}^{\infty} L_{0}\left(z(t), z^{\prime}(t)\right) d t, \quad|z(t)|<\eta,\left|z^{\prime}(t)\right|<\eta \text { a.e. } t>0,
$$

and $z(0)=y(T)$. Let $\tilde{y}:[0,+\infty[\rightarrow E$ be defined by

$$
\tilde{y}(t)= \begin{cases}y(t), & 0<t<T, \\ z(t-T), & T<t<+\infty .\end{cases}
$$

In consequence,

$$
\begin{aligned}
\Psi\left(x_{0}\right) & =\int_{0}^{\infty} L_{0}\left(x(t), x^{\prime}(t)\right) d t \leqslant \int_{0}^{\infty} L_{0}\left(\tilde{y}(t), \tilde{y}^{\prime}(t)\right) d t \\
& =\int_{0}^{T} L_{0}\left(y(t), y^{\prime}(t)\right) d t+\Psi(y(T)) .
\end{aligned}
$$

This demonstrates that

$$
\Psi\left(x_{0}\right) \leqslant \inf \left\{\int_{0}^{T} L\left(y(t), y^{\prime}(t)\right) d t+\Psi(y(T)) ; y(0)=x_{0}\right\}
$$

and

$$
\Psi(x(T)) \geqslant \int_{T}^{\infty} L_{0}\left(x(t), x^{\prime}(t)\right) d t .
$$

On the other hand, by definition of $\Psi(x(T))$ it follows that

$$
\Psi(x(T)) \leqslant \int_{0}^{\infty} L_{0}\left(y(t), y^{\prime}(t)\right) d t
$$

for all absolutely continuous arcs $y(t):[0,+\infty[\rightarrow E$ satisfying $|y(t)|<\eta$, $\left|y^{\prime}(t)\right| \leqslant \eta$ a.e. $t>0$ and $y(0)=x(T)$. In particular, for $y(t)=x(t+T)$ we have

$$
\Psi(x(T))<\int_{T}^{\infty} L\left(x(t), x^{\prime}(t)\right) d t .
$$

Combined with (2.22) and (2.23), this yields (2.21), thereby proving the lemma.

3. Proof of Theorem 1. We begin with the construction of the neighborhood $U$ specified in Theorem 1. Let $\partial L_{0}: E \times E \rightarrow E \times E$ be the subdifferential of $L_{0}$. It follows by Lemma 1 (part (2.4)) that the operator (multivalued) $\partial L_{0}$ is locally bounded on $\Sigma_{\delta}=\{(x, u) \in E \times E ;|x|<\delta,|u|<\delta\}$ where $0<\delta<$ $\eta$ (in the sequel $\delta$ will be chosen as in Lemma 2). Redefining $\delta$ if necessary, we are therefore justified in assuming from now on that

$$
\begin{array}{r}
\left\|\partial L_{0}(x, u)\right\|=\sup \left\{\left|y_{1}\right|+\left|y_{2}\right| ;\left(y_{1}, y_{2}\right) \in \partial L_{0}(x, u)\right\}<C_{0} \\
\text { for all }(x, u) \in \Sigma_{\delta} .
\end{array}
$$

On the other hand, by Lemma 1, part (2.5), we have

$$
L_{0}(0, u) /|u|<\delta / 2 \text { for all }|u|<\vartheta \text {. }
$$


We choose $\sigma$ in such a way that

$$
0<\sigma<\vartheta \delta / C_{0}<\delta
$$

where $C_{0}$ is the constant arising in (3.1). Let $\rho$ be the positive number which corresponds to $\sigma$ in virtue of Lemma 4 and let

$$
U=\left\{x_{0} \in E ; \Psi\left(x_{0}\right)<\rho\right\} .
$$

Thus, $U$ is an open neighborhood of 0 and it is contained in the ball $S_{\sigma}=\left\{x_{0} \in E ;\left|x_{0}\right| \leqslant \sigma\right\}$.

Let $L_{\lambda}: E \times E \rightarrow\left[0,+\infty\left[\right.\right.$ and $\Psi_{\lambda}: E \rightarrow[0,+\infty[$ be the convex functions defined by

$$
\begin{aligned}
L_{\lambda}(x, u) & =\inf \left\{\frac{|x-y|^{2}+|u-v|^{2}}{2 \lambda}+L(y, v) ; y \in E, v \in E\right\}, \\
\Psi_{\lambda}(x) & =\inf \left\{|x-y|^{2} / 2 \lambda+\Psi(y) ; y \in E\right\} .
\end{aligned}
$$

We recall that these functions are Fréchet differentiable on $E \times E$ and $E$, respectively. Moreover, one has (see [4, p. 39])

$$
L_{\lambda}(x, u) \leqslant L(x, u), \quad \Psi_{\lambda}(x)<\Psi(x) \text { for all } \lambda>0
$$

and

$$
\lim _{\lambda \rightarrow 0} L_{\lambda}(x, u)=L(x, u), \quad \lim _{\lambda \rightarrow 0} \Psi_{\lambda}(x)=\Psi(x)
$$

for all $(x, u) \in E \times E$ and $x \in E$. Other properties of these functions may be found in the above quoted book (see also [2]).

Let $x_{0} \in U$ be fixed. Then for every $\lambda>0$ and $T>0$ we consider the optimization problem

$$
\begin{aligned}
\inf \left\{\int_{0}^{T} L_{\lambda}\left(y(t), y^{\prime}(t)\right) d t+\frac{1}{2} \int_{0}^{T}\right. & \left|y^{\prime}(t)-x^{\prime}(t)\right|^{2} d t \\
& \left.+\Psi_{\lambda}(y(T)) ; y(0)=x_{0}\right\}
\end{aligned}
$$

where $x$ is the unique arc which yields the minimum in (2.13) (see Lemma 3). Obviously (3.6) admits a unique solution $x_{\lambda}:[0, T] \rightarrow E$. Moreover, it follows by a standard argument that for each $\lambda>0$ there exists a function $p_{\lambda}$ : $[0, T] \rightarrow E$ which satisfies together with $x_{\lambda}$ the Euler-Lagrange equations

$$
\left.\left(p_{\lambda}^{\prime}(t), p_{\lambda}(t)+x^{\prime}(t)-x_{\lambda}^{\prime}(t)\right)=\partial L_{\lambda}\left(x_{\lambda}(t), x_{\lambda}^{\prime}(t)\right) \text { a.e. } t \in\right] 0, T[
$$

and transversality conditions

$$
p_{\lambda}(T)=-\partial \Psi_{\lambda}\left(x_{\lambda}(T)\right), \quad x_{\lambda}(0)=x_{0} .
$$

The next thing to note is that (3.7) yields (by definition of $\partial L_{\lambda}$ ) 


$$
\begin{aligned}
\left(p_{\lambda}(t), x_{\lambda}(t)-\right. & x(t))+\left(p_{\lambda}^{\prime}(t), x_{\lambda}^{\prime}(t)-x^{\prime}(t)\right)-\left|x_{\lambda}^{\prime}(t)-x^{\prime}(t)\right|^{2} \\
& \left.\geqslant L_{\lambda}\left(x_{\lambda}(t), x_{\lambda}^{\prime}(t)\right)-L_{\lambda}\left(x(t), x^{\prime}(t)\right), \text { a.e. } t \in\right] 0, T[
\end{aligned}
$$

and, therefore,

$$
\begin{aligned}
\int_{0}^{T}\left|x_{\lambda}^{\prime}(t)-x^{\prime}(t)\right|^{2} d t< & \int_{0}^{T} L\left(x(t), x^{\prime}(t)\right) d t+\Psi(x(T)) \\
& -\int_{0}^{T} L_{\lambda}\left(x_{\lambda}(t), x_{\lambda}^{\prime}(t)\right) d t-\Psi_{\lambda}\left(x_{\lambda}(T)\right)
\end{aligned}
$$

because $\Psi_{\lambda}(y)<\Psi(y)$ and $L_{\lambda}\left(y_{1}, y_{2}\right)<L\left(y_{1}, y_{2}\right)$ for all $\lambda>0$ and $y, y_{1}, y_{2}$ in $E$. Thus proceeding as in [3] we deduce from (3.9) that

$$
\begin{array}{r}
\underset{\lambda \rightarrow 0}{\limsup }\left(\int_{0}^{T} L_{\lambda}\left(x_{\lambda}(t), x_{\lambda}^{\prime}(t)\right) d t+\Psi_{\lambda}\left(x_{\lambda}(T)\right)\right) \\
>\int_{0}^{T} L\left(y(t), y^{\prime}(t)\right) d t+\Psi(y(T))
\end{array}
$$

where $y(t)$ is an absolutely continuous arc on $[0, T]$ satisfying $y(0)=x_{0}$. Substituting the latter in (3.9) and applying Lemma 5, one sees that

$$
\lim _{\lambda \rightarrow 0} x_{\lambda}^{\prime}(t)=x^{\prime}(t) \text { strongly in } L^{2}(0, T ; E)
$$

and therefore

$$
\lim _{\lambda \rightarrow 0} x_{\lambda}(t)=x(t) \text { uniformly on }[0, T] .
$$

Returning to (2.21) in Lemma 5 we observe it implies that

$$
\frac{d}{d t} \Psi(x(t))=-L_{0}\left(x(t), x^{\prime}(t)\right)<0, \text { a.e. } t>0,
$$

and, hence, by (3.4), that

$$
x(t) \in U \text { for every } t>0 .
$$

On the other hand we have by definition of $\partial \Psi_{\lambda}$ that

$$
\left(\partial \Psi_{\lambda}\left(x_{\lambda}(T)\right), x_{\lambda}(T)-x_{0}-\gamma w\right)>\Psi_{\lambda}\left(x_{\lambda}(T)\right)-\Psi_{\lambda}\left(x_{0}+\gamma w\right),
$$

while by (3.7) it is also true that

$$
\begin{aligned}
\left(p_{\lambda}^{\prime}(t), x_{\lambda}(t)-x_{0}\right) & +\left(p_{\lambda}(t)+x^{\prime}(t)-x_{\lambda}^{\prime}(t), x_{\lambda}^{\prime}(t)\right) \\
& \left.>L_{\lambda}\left(x_{\lambda}(t), x_{\lambda}^{\prime}(t)\right)-L_{\lambda}\left(x_{0}, 0\right), \text { a.e. } t \in\right] 0, T[
\end{aligned}
$$

and, therefore,

$$
\left(p_{\lambda}(T), x_{\lambda}(T)-x_{0}\right)>\int_{0}^{T}\left(x_{\lambda}^{\prime}(t), x_{\lambda}^{\prime}(t)-x^{\prime}\right) d t-T L\left(x_{0}, 0\right) .
$$

Adding these inequalities and remembering transversality conditions (3.8) we obtain

$$
\gamma\left|\partial \Psi_{\lambda}\left(x_{\lambda}(T)\right)\right|<\Psi_{\lambda}\left(x_{0}+\gamma w\right)+C \text { for all } \lambda>0 \text { and }|w|=1 .
$$


But in view of Lemma 3 the function $\Psi$ is locally bounded on $S_{\eta}$. Hence for $\gamma$ sufficiently small, we have

$$
\Psi_{\lambda}\left(x_{0}+\gamma w\right) \leqslant \Psi\left(x_{0}+\gamma w\right) \leqslant C \text { for }|w|=1 \text { and } \lambda>0 .
$$

Thus

$$
\left|\partial \Psi_{\lambda}\left(x_{\lambda}(T)\right)\right| \leqslant C \text { for all } \lambda>0 .
$$

Now using once again (3.7) and definition of $\partial L_{\lambda}$ we get

$$
\begin{aligned}
\left(p_{\lambda}^{\prime}(t), x_{\lambda}(t)-\delta w\right)+\left(p_{\lambda}(t)+x^{\prime}(t)-x_{\lambda}^{\prime}(t), x_{\lambda}^{\prime}(t)\right) \\
\quad \geqslant L_{\lambda}\left(x_{\lambda}(t), x_{\lambda}^{\prime}(t)\right)-L_{\lambda}(\delta w, 0) \text { for }|w|=1 .
\end{aligned}
$$

Integrating over $[0, T]$ the latter inequality implies, via Lemma 1 ,

$$
\int_{0}^{T}\left|p_{\lambda}^{\prime}(t)\right| d t<C
$$

and therefore $\left|p_{\lambda}(t)\right| \leqslant C$ for all $t \in[0, T]$ and $\lambda>0$. Moreover, we have

$$
\left.\delta\left|p_{\lambda}^{\prime}(t)\right|<\left|x_{\lambda}(t)\right|\left|p_{\lambda}^{\prime}(t)\right|+C\left|x_{\lambda}^{\prime}(t)\right|+\left|x_{\lambda}^{\prime}(t)\right|\left|x^{\prime}(t)\right| \text {, a.e. } t \in\right] 0, T[\text {. }
$$

Since $U \subset S_{\sigma}$ it follows from (3.3), (3.10) and (3.12) that there exists $0<\delta^{\prime}<\delta$ such that $\left|x_{\lambda}(t)\right| \leqslant \delta^{\prime}$ for all $t \in[0, T]$ and sufficiently small $\lambda$. This fact, combined with the above inequality, yields

$$
\int_{0}^{T}\left|p_{\lambda}^{\prime}(t)\right|^{2} d t<C \text { for all sufficiently small } \lambda>0 .
$$

Thus extracting a subsequence, if necessary, we may assume that for $\lambda \rightarrow 0$,

$$
\begin{array}{ll}
p_{\lambda}^{\prime} \rightarrow p_{T}^{\prime} & \text { weakly in } L^{2}(0, T ; E), \\
p_{\lambda} \rightarrow p_{T} & \text { weakly-star in } L^{\infty}(0, T ; E) .
\end{array}
$$

Since $\partial L$ (the subdifferential of $L$ ) is maximal monotone on $E \times E$ and $\dot{\partial} L_{\lambda}=\lambda^{-1}\left(I-(I+\lambda \partial L)^{-1}\right)$, it follows from (3.7), (3.9), (3.10), (3.14) and (3.15) that (see e.g. [2, p. 42])

$$
\left.\left(p_{T}^{\prime}(t), p_{T}(t)\right) \in \partial L\left(x(t), x^{\prime}(t)\right) \text { a.e. } t \in\right] 0, T[.
$$

Similarly, (3.14) implies in conjunction with (3.8) and (3.10) that

$$
p_{T}(T) \in-\partial \Psi(x(T))
$$

where consistent with the above notations $\partial \Psi: E \rightarrow E$ stands for the subdifferential of $\Psi$.

We claim that

$$
p_{T}(t) \in-\partial \Psi(x(t)) \text { for all } 0<t<T .
$$

Here is the argument. Let $h$ be arbitrary but fixed in $D(\Psi)$ and let $y$ : $\left[0, \infty\left[\rightarrow E\right.\right.$ be an absolutely continuous arc such that $y(0)=h$ and $\int_{0}^{\infty} L(y(t)$, $\left.y^{\prime}(t)\right) d t=\Psi(h)$ or, equivalently (see (2.21)), 


$$
\Psi(h)=\int_{0}^{t} L\left(y(t), y^{\prime}(t)\right) d t+\Psi(y(t)) \text { for all } t>0 .
$$

We fix $t$ in $[0, T]$ and set $z(s)=y(s-t)$ for $s>t$. We have from (3.16) and definition of $\partial L$ that

$$
\begin{aligned}
\left(p_{T}^{\prime}(s), x(s)-z(s)\right)+\left(p_{T}(s), x^{\prime}(s)-z^{\prime}(s)\right) \\
\quad \geqslant L\left(x(s), x^{\prime}(s)\right)-L\left(z(s), z^{\prime}(s)\right) \text { a.e. } t<s<T .
\end{aligned}
$$

Then, by (2.21) and (3.17),

$$
-\left(p_{T}(t), x(t)-h\right) \geqslant \Psi(x(t))-\Psi(y(T))-\int_{t}^{T} L\left(y(s), y^{\prime}(s)\right) d s .
$$

From this and (3.19), it is clear that one will have

$$
-\left(p_{T}(t), x(t)-h\right) \geqslant \Psi(x(t))-\Psi(h) .
$$

(3.19) is thereby established because $h$ is arbitrary in $D(\Psi)$. The next thing to verify is that $p_{T}$ satisfies the estimate

$$
\left|p_{T}(t)\right|<\delta \text { for all } 0<t<T \text { and } T>0 .
$$

As noticed earlier, (2.21) implies that

$$
\frac{d}{d t} \Psi(x(t))=-L_{0}\left(x(t), x^{\prime}(t)\right) \text { a.e. } t>0
$$

while (3.18) yields

$$
\frac{d}{d t} \Psi(x(t))=-\left(p_{T}(t), x^{\prime}(t)\right) \text { a.e. } 0<t<T .
$$

Hence

$$
L_{0}\left(x(t), x^{\prime}(t)\right)=\left(p_{T}(t), x^{\prime}(t)\right) \text { a.e. } 0<t<T .
$$

Since $L=L_{0}$ on $\Sigma_{\eta}$, the latter equality in conjunction with (3.16) yields

$$
\left(p_{T}(t), u\right) \leqslant L_{0}(x(t), u) \text { for all }|u|<\eta \text { and } t \in[0, T] \text {. }
$$

Also by definition of $\partial L_{0}(x, u)$ we have

$$
L_{0}(x(t), u)<L_{0}(0, u)+(y, x(t)) \text { for }|u|<\eta
$$

where $(y, 0) \in \partial L_{0}(x(t), u)$. Then by (3.2), (3.3) and (3.21) we get (3.20) as claimed. By Lemma $2, H(x, p)=H_{1}(x, p)$ for $|x|<\delta$ and $|p|<\delta$. This easily implies that $\partial H(x, p)=\partial H_{1}(x, p)$ if $|x|<\delta$ and $|p|<\delta$. Since by (3.12) $|x(t)| \leqslant \sigma<\delta$, and $\left|p_{T}(t)\right|<\delta$ by (3.20), we may conclude that $\partial H_{1}\left(x(t), p_{T}(t)\right)=\partial H\left(x(t), p_{T}(t)\right)$ a.e. $\left.t \in\right] 0, T[$ for all $T>0$.

On the other hand, it is well known that (3.16) can be equivalently written as

$$
\left(p_{T}^{\prime}(t), x^{\prime}(t)\right) \in \partial H_{1}\left(x(t), p_{T}(t)\right) \text { a.e. } 0<t<T .
$$

Comparison of this relation with (3.22) shows that $x(t)$ and $p_{T}(t)$ satisfy 


$$
\begin{array}{ll}
x^{\prime}(t) \in \partial_{p} H\left(x(t), p_{T}(t)\right) & \text { a.e. } 0<t<T, \\
p_{T}^{\prime}(t) \in-\partial_{x} H\left(x(t), p_{T}(t)\right) & \text { a.e. } 0<t<T .
\end{array}
$$

Recall that by assumption (b) the function $p \rightarrow H(x, p)$ is strictly convex in the neighborhood $S_{\eta}$ of 0 . In particular, this fact implies that if $\partial_{p} H(x, p)$ $\cap \partial_{q} H(x, q) \neq \varnothing$ then $p=q$, which implies the uniqueness of function $p_{T}$ satisfying, together with $x$, (3.23). In particular, it follows that $p_{T}(t)=p_{T^{\prime}}(t)$ over $[0, T]$ if $T^{\prime} \geqslant T$. Thus the function $p:[0, \infty[\rightarrow E$ defined by

$$
p(t)=p_{T}(t) \text { for } 0 \leqslant t \leqslant T
$$

satisfies with $x(t)$ the Hamiltonian system (1.1), i.e.,

$$
\begin{array}{ll}
x^{\prime}(t) \in \partial_{p} H(x(t), p(t)), & \text { a.e. } t>0, \\
p^{\prime}(t) \in-\partial_{x} H(x(t), p(t)), & \text { a.e. } t>0 .
\end{array}
$$

Moreover, one has (see (3.18))

$$
p(t) \in-\partial \Psi(x(t)) \text { for all } t \geqslant 0 .
$$

Coming back to (3.21), we obtain

$$
|p(t)| \leqslant C_{0}|x(t)| /|u|+L_{0}(0, u) /|u| \text { for all } t \geqslant 0 \text { and }|u| \leqslant \delta .
$$

Inasmuch as $\lim _{t \rightarrow \infty} x(t)=0$ and $L_{0}(0, u) /|u| \rightarrow 0$ as $|u| \rightarrow 0$, we deduce that

$$
\lim _{t \rightarrow \infty} p(t)=0 \text {. }
$$

Summarising at this point, we have shown that for every $x_{0} \in U$ there exists a unique solution $(x(t), p(t))$ to system (1.1) satisfying (1.8) and $x(0)=x_{0}$. (The uniqueness of $x$ follows as seen earlier from the extremal property of this arc and the strict convexity of $H$ as a function of $x$.)

This simply says that the operator $\Gamma$ defined in $\$ 1$ is defined on all of $U$. Moreover, (3.25) yields

$$
-\Gamma x_{0} \in \partial \Psi\left(x_{0}\right) \text { for all } x_{0} \in U .
$$

It is clear from (3.12) that the solution $(x(t), p(t))$ to (1.11) emanating from points of $\Gamma \cap(U \times \Gamma(U))$ remains in this set. Thus part (iii) of the theorem was established with $U$ constructed as above and $V=\Gamma(U)$. As remarked earlier the strict convexity of $H(x, p)$ in $p$ implies the uniqueness of $p(t)$ in (1.1). In other words, for each $x_{0} \in U$, there is no more than one $p_{0} \in E$ such that $p_{0} \in \Gamma x_{0}$ (i.e. $\Gamma$ is single valued on $U$ ). Similarly, the strict concavity of $H(x, p)$ in $x$ implies that for each $p_{0} \in V$, there is no more than one $x_{0} \in U$ such that $p_{0} \in \Gamma x_{0}$. Thus $\Gamma$ is single valued and one-to-one from $U$ onto $V$.

It should be observed also that (3.26) for $t=0$ gives

$$
|p(0)|=\left|\Gamma x_{0}\right| \leqslant C_{0}\left|x_{0}\right| /|u|+L_{0}(0, u) /|u| \text { for }|u|<\delta .
$$

Using once again Lemma 1, part (2.5), we deduce that, no matter what ball $S_{\sigma}=\{p \in E ;|p| \leqslant \sigma\}$ is specified, we can choose $U$ as above such that 
$V=\Gamma(U) \subset S_{o}$. In particular, this fact shows that the neighborhood $U \times V$ can be chosen arbitrarily small.

Next we shall prove that $\Gamma$ is demicontinuous on $U$, i.e. $\Gamma$ is continuous from the strong topology of $E$ to weak topology of $E$. Let $\left\{x_{0}^{n}\right\} \subset E$ be strongly convergent to $x_{0}$ as $n \rightarrow \infty$ and let $p_{0}^{n}=\Gamma x_{0}^{n}$. By definition of $\Gamma$ there exists a pair $\left(x_{n}(t), p_{n}(t)\right)$ satisfying over $[0,+\infty[$ the system

$$
\begin{array}{ll}
x_{n}^{\prime}(t) \in \partial_{p} H\left(x_{n}(t), p_{n}(t)\right), & \text { a.e. } t>0, \\
p_{n}^{\prime}(t) \in-\partial_{x} H\left(x_{n}(t), p_{n}(t)\right), & \text { a.e. } t>0,
\end{array}
$$

with initial conditions

$$
x_{n}(0)=x_{0}^{n}, \quad p_{n}(0)=p_{0}^{n},
$$

and

$$
\lim _{t \rightarrow \infty} x_{n}(t)=\lim _{t \rightarrow \infty} p_{n}(t)=0 \text { for all } n .
$$

Moreover, (3.25) shows that

$$
p_{n}(t) \in-\partial \Psi\left(x_{n}(t)\right) \text { for all } t>0 .
$$

Since $\partial \Psi$ is monotone on $E \times E$, the latter implies that for every $T>0$,

$$
\begin{aligned}
& \limsup _{m, n \rightarrow \infty} \int_{0}^{T}\left(\left(x_{n}^{\prime}(t)\right.\right.\left.-x_{m}^{\prime}(t), p_{n}(t)-p_{m}(t)\right) \\
&\left.+\left(p_{m}^{\prime}(t)-p^{\prime}(t), x_{n}(t)-x_{m}(t)\right)\right) d t \\
&<-\lim _{m, n \rightarrow \infty}\left(p_{n}^{0}-p_{m}^{0}, x_{n}^{0}-x_{m}^{0}\right)=0 .
\end{aligned}
$$

Since $\left(x_{n}, p_{n}\right)$ all belong to $\Sigma_{\delta}$, and by (1.6) $\partial H$ is bounded on $\Sigma_{\eta} \supseteq \Sigma_{\delta}$, we may suppose that for $n \rightarrow \infty$, we have

$$
\begin{aligned}
& x_{n}^{\prime} \rightarrow \tilde{x}^{\prime} \quad \text { weakly in } L^{2}(0, T ; E), \\
& p_{n}^{\prime} \rightarrow \tilde{p}^{\prime} \quad \text { weakly in } L^{2}(0, T ; E),
\end{aligned}
$$

respectively,

$$
\begin{array}{ll}
x_{n} \rightarrow \tilde{x} & \text { weakly in } L^{2}(0, T ; E), \\
p_{n} \rightarrow \tilde{p} \quad \text { weakly in } L^{2}(0, T ; E) .
\end{array}
$$

Denote by $\Delta$ the realization of the operator $\partial H$ from the space $\left(L^{2}(0, T ; E)\right.$ $\left.\times L^{2}(0, T ; E)\right)$ into itself. In other words,

$$
\Lambda(y, q)(t)=\partial H(y(t), q(t)) \text { a.e. } t>0 .
$$

This operator remains maximal monotone so that according to a well-known result (see e.g. [2, p. 42]), relations (3.28), (3.32), (3.33) and (3.34) give

$$
\left.\left(\tilde{p}^{\prime}(t), \tilde{x}^{\prime}(t)\right) \in \partial H(\tilde{x}(t), \tilde{p}(t)), \text { a.e. } t \in\right] 0, T[\text {. }
$$


Clearly

$$
\tilde{x}(0)=x_{0}, \quad \tilde{p}(0)=p_{0}
$$

and

$$
\lim _{n \rightarrow \infty} x_{n}(T)=\tilde{x}(T), \quad \lim _{n \rightarrow \infty} p_{n}(T)=\tilde{p}(T)
$$

in the weak topology of $E$. Again using (3.28) and the monotonicity of $\partial H$ we get

$$
\begin{aligned}
\liminf _{m, n \rightarrow \infty}( & \left.x_{n}(T)-x_{m}(T), p_{n}(T)-p_{m}(T)\right) \\
& \geqslant \liminf _{m, n \rightarrow \infty}\left(x_{n}^{0}-x_{m}^{0}, p_{m}^{0}-p_{n}^{0}\right)=0 .
\end{aligned}
$$

Since $\partial \Psi$ is maximal monotone from $E$ into itself the latter inequality combined with (3.31) and (3.37) yields

$$
\tilde{p}(T) \in-\partial \Psi(\tilde{x}(T)) .
$$

It is well known that the Hamiltonian equations (3.35) together with the transversality condition (3.38) imply the solution of the Bolza problem (see [3] for a treatment of more general problems of this type in the Hilbert space general setting)

$$
\inf \left\{\int_{0}^{T} L\left(y, y^{\prime}\right) d t+\Psi(y(T)) ; y(0)=x_{0}\right\} .
$$

But as seen in Lemma 5 the unique solution of (3.39) coincides over $[0, T]$ with the function $x:\left[0,+\infty\left[\rightarrow E\right.\right.$ which yields the infimum in $\Psi\left(x_{0}\right)$. Since $x_{0} \in U$, there exists an absolutely continuous function $p(t):[0,+\infty[\rightarrow E$ satisfying, together with $x(t)$, the Hamiltonian system (1.1) and relation (1.8). Thus as observed earlier, (3.35) implies that $p(t)=\tilde{p}(t)$ over $[0, T]$. In particular, it follows that $p_{0}=p(0)=\Gamma x_{0}$. Hence we have shown that $\lim _{n \rightarrow \infty} \Gamma x_{0}^{n}=\Gamma x_{0}$ in the weak topology of $E$.

Now we shall prove that $\partial \Psi$ is single valued and agrees with $-\Gamma$ on $U$. Let $x_{0}$ be arbitrary but fixed in $U$ and let $y_{0}$ be any element of $\partial \Psi\left(x_{0}\right)$. Since $-\Gamma \subset \partial \Psi$ on $U$ and $\partial \Psi$ is monotone we have

$$
-\left(\Gamma u+y_{0}, u-x_{0}\right)>0 \text { for all } u \in U .
$$

Let $v$ be arbitrary in $E$ and let $u_{t}=t x_{0}+(1-t) v, 0<t<1$. For $1-t$ sufficiently small, $u_{t} \in U$ and, therefore, (3.40) yields

$$
\lim _{t \rightarrow 1}\left(\Gamma u_{t}+y_{0}, v-x_{0}\right)<0 .
$$

Since $\Gamma$ is demicontinuous and $v$ is arbitrary we have immediately that $\Gamma x_{0}=-y_{0}$ as claimed. Note that the facts that $\partial \Psi$ is single valued and $\Psi$ is continuous on $U$ imply by a well-known result that $\Psi$ is Gâteaux differentiable on $U$ and its Gâteaux differential $\nabla \Psi$ agrees with $\partial \Psi$ on $U$.

Now we shall prove that $V=\Gamma(U)$ is a neighborhood of the origin. Let $y$ 
be arbitrary but fixed in $E$. Inasmuch as $\partial \Psi: E \rightarrow E$ is maximal monotone and its domain is bounded (in fact it is contained in $D(\Psi)=S_{\eta}$ ), the equation $\partial \Psi(x) \ni y$ has at least one solution $x_{0} \in S_{\eta}$. Multiplying each side of the preceding equation by $x_{0}$ one has that

$$
\Psi\left(x_{0}\right) \leqslant\left|x_{0}\right||y| \leqslant \eta|y| \text {. }
$$

It now follows directly from Lemma 4 that $x_{0} \in U$ if $|y|<\gamma$ where $\gamma$ is sufficiently small. In other words we have shown that $V=\Gamma(U)$ contains the open ball $S_{\gamma}=\left\{x_{0} \in E ;\left|x_{0}\right|<\gamma\right\}$ as claimed.

All that is left to prove in Theorem 1 are parts (iv) and (v). Fix any $\left(x_{0}, p_{0}\right) \in \Gamma$. Let $x$ and $p$ denote the corresponding unique solution to (1.1) over [0, $+\infty$ [ satisfying (1.7) and (1.8). It follows by (1.6) that $H(x(t), p(t))$ is an absolutely continuous function of $t \in[0,+\infty[$, and that

$$
\begin{aligned}
\frac{d}{d t} H(x(t), p(t))=( & \left.x\left(t^{\prime}\right), \partial_{x} H(s(t), p(t))\right) \\
& +\left(p^{\prime}(t), \partial_{p} H(x(t), p(t))\right)=0, \text { a.e. } t>0 .
\end{aligned}
$$

(The proof is essentially the same as that of Theorem 3 in [6].) We may therefore conclude that

$$
\frac{d}{d t} H(x(t), p(t))=0 \quad \text { a.e. } t>0,
$$

thereby proving (1.10).

It remains to prove part (v). Let $(\tilde{x}(t), \tilde{p}(t))$ be a solution to (1.1) over $[0,+\infty[$ satisfying

$$
\tilde{x}(t) \in U, \tilde{p}(t) \in V \quad \text { for all } t \geqslant 0 .
$$

Thus by definition of $\partial H$, we have

$$
\frac{d}{d t}(\tilde{x}(t), \tilde{p}(t)) \geqslant H(0, \tilde{p}(t))-H(\tilde{x}(t), 0) \text { a.e. } t>0 .
$$

In particular, this inequality shows that $\lim _{t \rightarrow+\infty}(\tilde{x}(t), \tilde{p}(t))$ exists and therefore the functions $H(0, \tilde{p}(t))$ and $-H(\tilde{x}(t), 0)$ are summable over $[0 ;+\infty[$. Since $\tilde{x}(t)$ and $\tilde{p}(t)$ are uniformly continuous over $\left[0,+\infty\left[\right.\right.$ (because $x^{\prime}(t)$ and $p^{\prime}(t)$ are bounded on $[0,+\infty[$ by virtue of $(1.6))$ we may deduce that

$$
\lim _{t \rightarrow+\infty} H(0, \tilde{p}(t))=\lim _{t \rightarrow+\infty} H(\tilde{x}(t), 0)=0 .
$$

Therefore, by Lemma 1 (parts (2.6) and (2.7)) we infer that $\lim _{t \rightarrow+\infty} \tilde{x}(t)=$ $\lim _{t \rightarrow+\infty} \tilde{p}(t)=0$, thereby proving that $(x(t), p(t)) \in \Gamma$ for all $t \geqslant 0$ as claimed. This completes the proof of the theorem.

\section{REFERENCES}

1. V. Barbu, Convex control problem of Bolza in Hilbert space, SIAM J. Control 13 (1975), 754-771.

2. Nonlinear semigroups and evolution equations in Banach spaces, Noordhoff, 
Groningen, Publishing House of Romanian Academy, 1976.

3. Appl. 57 (1976), 502-528.

4. H. Brézis, Opérateurs maximaux monotones et semi-groupes de contractions dans les espaces de Hilbert, Math. Studies, no. 5, North-Holland, Amsterdam, 1973.

5. J. Moreau, Fonctionnelles convexes, Séminaire sur les équations aux dérivées partielles, College de France, 1966-1967.

6. R. T. Rockafellar, Generalized Hamiltonian equations for convex problems of Lagrange, Pacific J. Math. 33 (1970), 441-472.

7. , Saddle-points and convex analysis, Differential Games and Related Topics, North-Holland, Amsterdam, 1971, pp. 109-128.

8. , Monotone operators associated with saddle functions and minimax problems, Proc. Sympos. Pure Math., vol. 18, part 1, Amer. Math. Soc., Providence, R.I., 1970, pp. 241-251.

9. - Saddle points of Hamiltonian systems in convex problems of Lagrange, J. Optimization Theory Appl. 12 (1973), 367-390.

10. , Saddle points of Hamiltonian systems in convex Lagrange problems having a nonzero discount rate, J. Economic Theory 12 (1976), 71-113.

FACUlty OF MATHEMATICS, UNIVERSITY OF IASI, IASI, ROMANIA 